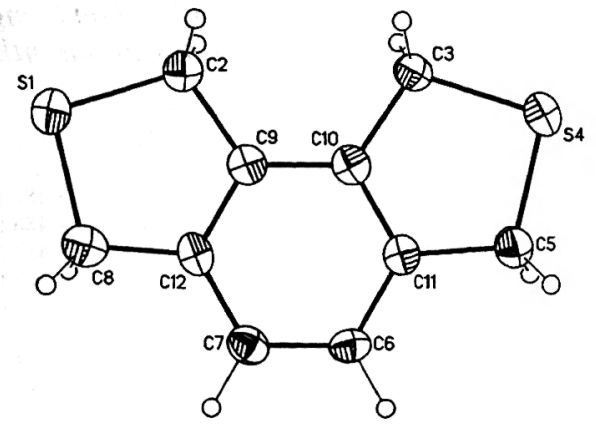

(I)

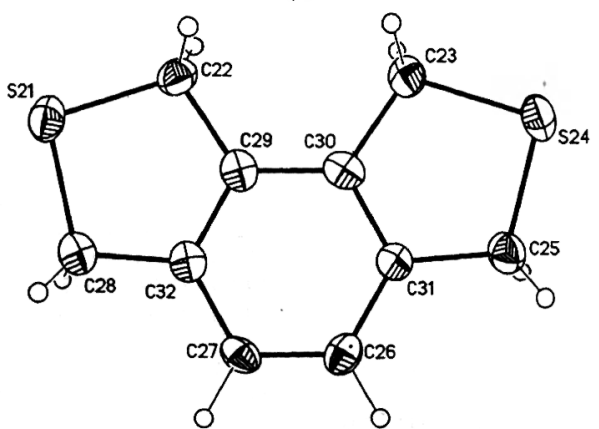

(II)

Fig. 1. Perspective view of molecules (I) and (II) showing the atomic labelling. Ellipsoids are drawn to include $50 \%$ probability for non-H atoms.

The packing is determined by the formation of intermolecular $\mathrm{C}-\mathrm{H} \cdots \mathrm{S}$ hydrogen bonds with $\mathrm{H} \cdots \mathrm{S}$ distances of $2.90 \pm 0.06 \AA$ (mean value) and $\mathrm{C}-\mathrm{H} \cdots \mathrm{S}$ angles of $146 \pm 10^{\circ}$ (mean value). Furthermore, intermolecular $\mathrm{S} \cdots \mathrm{S}$ distances of $3.64 \AA$, shorter than the sum of the van der Waals radii, were observed.
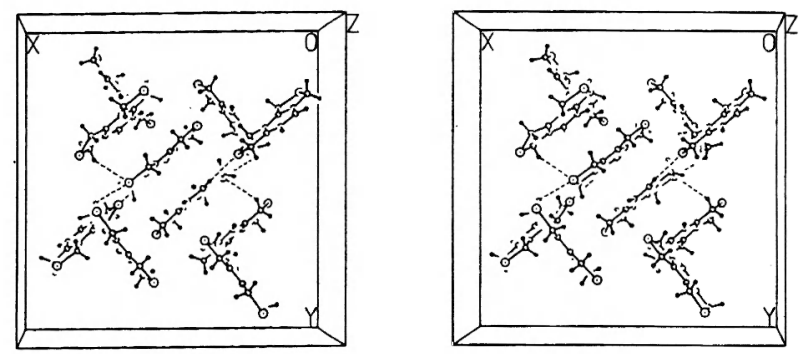

Fig. 2. Stereoview showing the crystal packing along the $z$ axis.

\section{References}

Bacon, G. E., Curry, N. A. \& Wilson, S. A. (1964). Proc. $R$. Soc. London Ser. A, 279, 98-110.

Baldridge, K. K. \& Siegel, J. S. (1992). J. Am. Chem. Soc. 114, 9583-9587.

Brett, W. A., Rademacher, P. \& Boese, R. (1993). Acta Cryst. C49, 1564-1566.

Cromer, D. T. \& Liberman, D. (1970). J. Chem. Phys. 53, 1891-1894.

Cromer, D. T. \& ManN, J. B. (1968). Acta Cryst. A24, 321324.

Faust, R., Glendening, E. D., Streitwieser, A. \& Vollhardt, K. P. C. (1992). J. Am. Chem. Soc. 114, 8263-8268.

Giovannini, E. \& Vuilleumier, H. (1977). Helv. Chim. Acta, 60, $1452-1455$.

Kay, M. I., OKayA, Y. \& Cox, D. E. (1971). Acta Cryst. B27, 26-33.

Mills, W. H. \& Nixon, I. G. (1930). J. Chem. Soc. pp. 25102524.

RADEMACHER, P. (1987). Strukturen organischer Moleküle. Weinheim: VCH

SHELDRICK, G. M. (1988). SHELXTL-Plus88. Structure Determination Software Programs. Nicolet Instrument Corporation, Madison, Wisconsin, USA.

Stanger, A. (1991). J. Am. Chem. Soc. 113, 8277-8280, and references therein.

Acta Cryst. (1993). C49, 1568-1571

\title{
Structure of the Product from a Novel Cyclization Reaction Involving a C(6)-Substituted Uridine Analog
}

\author{
By Binghe Wang, ${ }^{*}$ Fusao Takusagawa, Mathias P. Mertes $\dagger$ and Kristin Bowman-James $\ddagger$
} Departments of Chemistry and Medicinal Chemistry, University of Kansas, Lawrence, Kansas 66045, USA

(Received 10 January 1992; accepted 23 April 1993)

Abstract. $\quad 5,6,7,7 \mathrm{a}, 9,10,14 \mathrm{~b}, 14 \mathrm{c}-$ Octahydro-4-[2,3-O(1-methylethylidene)- $\beta$-D-ribofuranosyl]cyclopenta-

\footnotetext{
* Current address: Department of Pharmaceutical Chemistry, University of Kansas, Lawrence, KS 66045, USA

+ Deceased.

$\ddagger$ To whom correspondence should be addressed.

C 1993 International Union of Crystallography Printed in Great Britain - all rights reserved
}

[4,5]pyrimido $\left[5^{\prime}, 4^{\prime}: 3,4\right]$ pyrrolo[2,1-a]isoquinoline$1,3(2 H, 4 H)$-dione, $\mathrm{C}_{25} \mathrm{H}_{31} \mathrm{~N}_{3} \mathrm{O}_{6}, M_{r}=469.54$, tetragonal, $P 4_{3} 22_{1} 2, a=12.577$ (2), $b=12.577$ (2), $c=$ $29.893(4) \AA, \quad V=4729(1) \AA^{3}, \quad Z=8, \quad D_{x}=$ $1.319 \mathrm{~g} \mathrm{~cm}^{-3}, \lambda(\mathrm{Cu} K \alpha)=1.5418 \AA, \mu=7.9 \mathrm{~cm}^{-1}$, $F(000)=2000, \quad T=298 \mathrm{~K}$, final $R=0.038, w R=$ 
0.064 for all 1884 independent reflections and 432 variables. The crystal structure shows a syn conformation around the $\mathrm{N}(1)-\mathrm{C}\left(1^{\prime}\right)$ single bond, consistent with other $\mathrm{C}(6)$-substituted uridine analogs, and an unusual $\mathrm{O}\left(1^{\prime}\right)$ endo conformation of the ribose ring. The stereochemistries of the three newly created chiral centers are $14 \mathrm{c} R, 4 \mathrm{a} R, 7 \mathrm{a} S$.

Introduction. During the synthesis of $\mathrm{C}(6)$-substituted uridine analogs, a novel 1,3-dipolar cycloaddition reaction involving the $\mathrm{C}(5)=\mathrm{C}(6)$ double bond was discovered (Wang, Mertes, Mertes \& Takusagawa, 1990). From this reaction, out of the four expected diastereomeric products, only two, (1) and (2), were formed and isolated in a ratio of 1:3.6. The ${ }^{1} \mathrm{H}$ NMR spectrum of compound (1) exhibited an unusual chemical shift for the $\mathrm{C}\left(2^{\prime}\right)$ proton, which indicates that the isopropylideneribose ring is twisted from a position normally seen for $\mathrm{C}(6)$-substituted uridine analogs. The elucidation of the stereochemistry of this reaction and the underlying reason for the unusual chemical shift of the $C(2)$ proton was made on the basis of crystallographic studies of one of the products.

Experimental. The preparation of compounds (1) and (2) has been described (Wang et al., 1990).

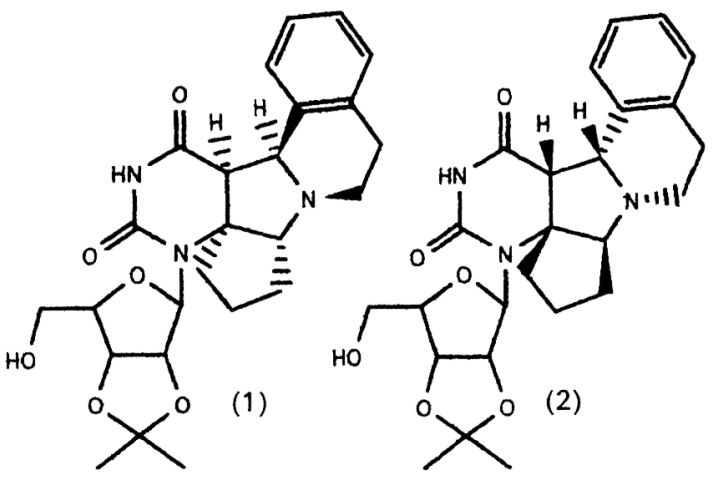

Compound (1) was crystallized from a mixture of methanol and methylene chloride by slowly evaporating the solvent at room temperature. A slightly yellow crystal, $0.2 \times 0.2 \times 0.4 \mathrm{~mm}$, was mounted on a glass fiber oriented approximately along [001]. Cell constants were determined using 15 centered reflections widely scattered throughout reciprocal space $\left(2 \theta>25^{\circ}\right)$. Preliminary counter data indicated a tetragonal system with systematic absences, $h=2 n$ in $h 00$ and $l=4 n$ in $00 l$, suggesting as possible space groups $P 4_{1} 2_{1} 2$ or $P 4_{3} 2_{1} 2$. The latter group was assigned by referring to the known configuration of the $\beta$-D-ribose moiety. One octant $(h=0-13, k=$ $0-13, l=0-32)$ of data out to $2 \theta=112.5^{\circ}$ were collected using a Syntex $P 2_{1}$ diffractometer $(\mathrm{Cu} K \alpha$,
Table 1. Fractional coordinates and equivalent isotropic thermal parameters $\left(\times 10^{2}\right)$ for non- $\mathrm{H}$ atoms

\begin{tabular}{lcccc}
\multicolumn{5}{c}{$U_{\text {eq }}=(1 / 3) \sum_{i} \sum_{j} U_{i j} a_{i}^{*} a_{j}^{*} \mathbf{a}_{i} \cdot \mathbf{a}_{j}}$. \\
& $x$ & $y$ & $z$ & \\
$\mathrm{~N}(1)$ & $0.2614(2)$ & $-0.0796(2)$ & $0.58827(9)$ & $U_{\text {eq }}\left(\AA^{2}\right)$ \\
$\mathrm{C}(2)$ & $0.3541(3)$ & $-0.1296(3)$ & $0.5999(1)$ & 4.08 \\
$\mathrm{~N}(3)$ & $0.3451(2)$ & $-0.2369(2)$ & $0.61185(9)$ & 4.09 \\
$\mathrm{C}(4)$ & $0.2544(3)$ & $-0.2876(3)$ & $0.6242(1)$ & 4.38 \\
$\mathrm{C}(5)$ & $0.1539(3)$ & $-0.2252(3)$ & $0.6200(1)$ & 4.42 \\
$\mathrm{C}(6)$ & $0.1588(3)$ & $-0.1354(3)$ & $0.5856(1)$ & 4.01 \\
$\mathrm{C}(7)$ & $0.1334(3)$ & $-0.1731(3)$ & $0.5380(1)$ & 4.77 \\
$\mathrm{C}(8)$ & $0.0127(3)$ & $-0.1702(4)$ & $0.5363(1)$ & 4.71 \\
$\mathrm{C}(9)$ & $-0.0155(3)$ & $-0.0678(3)$ & $0.5607(1)$ & 5.55 \\
$\mathrm{C}(10)$ & $0.0623(3)$ & $-0.0646(3)$ & $0.5998(1)$ & 4.93 \\
$\mathrm{~N}(11)$ & $0.0188(2)$ & $-0.1154(2)$ & $0.64071(8)$ & 3.94 \\
$\mathrm{C}(12)$ & $-0.0329(3)$ & $-0.0412(3)$ & $0.6717(1)$ & 3.72 \\
$\mathrm{C}(13)$ & $0.0438(3)$ & $0.0468(3)$ & $0.6875(1)$ & 4.61 \\
$\mathrm{C}(14)$ & $0.1498(3)$ & $0.0003(3)$ & $0.70035(9)$ & 4.75 \\
$\mathrm{C}(15)$ & $0.2222(3)$ & $0.0651(4)$ & $0.7239(1)$ & 4.64 \\
$\mathrm{C}(16)$ & $0.3187(4)$ & $0.0253(5)$ & $0.7384(1)$ & 5.55 \\
$\mathrm{C}(17)$ & $0.3439(3)$ & $-0.0807(4)$ & $0.7308(1)$ & 6.97 \\
$\mathrm{C}(18)$ & $0.2740(3)$ & $-0.1433(3)$ & $0.7072(1)$ & 6.32 \\
$\mathrm{C}(19)$ & $0.1782(3)$ & $-0.1035(3)$ & $0.69039(9)$ & 4.99 \\
$\mathrm{C}(20)$ & $0.1066(2)$ & $-0.1741(3)$ & $0.6625(1)$ & 4.24 \\
$\mathrm{O}(2)$ & $0.4409(2)$ & $-0.0882(2)$ & $0.59946(8)$ & 4.05 \\
$\mathrm{O}(4)$ & $0.2561(2)$ & $-0.3792(2)$ & $0.6373(1)$ & 5.29 \\
$\mathrm{C}\left(1^{\prime}\right)$ & $0.2684(3)$ & $0.0282(3)$ & $0.5709(1)$ & 6.32 \\
$\mathrm{C}\left(2^{\prime}\right)$ & $0.3067(3)$ & $0.1138(3)$ & $0.6036(1)$ & 4.19 \\
$\mathrm{C}\left(3^{\prime}\right)$ & $0.3750(3)$ & $0.1876(3)$ & $0.5757(1)$ & 4.62 \\
$\mathrm{C}\left(4^{\prime}\right)$ & $0.3829(3)$ & $0.1371(3)$ & $0.5293(1)$ & 5.36 \\
$\mathrm{C}\left(5^{\prime}\right)$ & $0.4963(4)$ & $0.1218(4)$ & $0.5130(1)$ & 5.05 \\
$\mathrm{C}\left(6^{\prime}\right)$ & $0.2477(4)$ & $0.2849(3)$ & $0.6121(1)$ & 6.45 \\
$\mathrm{C}\left(7^{\prime}\right)$ & $0.1541(6)$ & $0.3525(6)$ & $0.6006(3)$ & 6.35 \\
$\mathrm{C}\left(8^{\prime}\right)$ & $0.3020(7)$ & $0.3265(6)$ & $0.6524(2)$ & 9.84 \\
$\mathrm{O}\left(1^{\prime}\right)$ & $0.3384(2)$ & $0.0314(2)$ & $0.53342(7)$ & 9.50 \\
$\mathrm{O}\left(2^{\prime}\right)$ & $0.2185(2)$ & $0.1778(2)$ & $0.61695(9)$ & 4.74 \\
$\mathrm{O}\left(3^{\prime}\right)$ & $0.3166(3)$ & $0.2835(2)$ & $0.5742(1)$ & 6.37 \\
$\mathrm{O}\left(5^{\prime}\right)$ & $0.5617(2)$ & $0.0689(3)$ & $0.5450(1)$ & 7.90 \\
& & & & 7.40 \\
& & & &
\end{tabular}

graphite monochromator) with a $\theta-2 \theta$ scan mode (scan angle: $0.6894 \tan \theta+2.4^{\circ}$; scan speed: 1.5 to $20.0^{\circ} \mathrm{min}^{-1}$; background: $\frac{1}{6}$ of total scan at both edges). The total number of reflections measured was 3642. Lorentz and polarization factors were applied. Two standard reflections monitored every 100 measurements showed no significant decay of the crystal. The $\psi$-scan indicated no significant absorption effect, so no absorption correction was applied. After merging equivalent reflections, 1884 independent $F_{0}{ }^{2}$ data were obtained with an $R_{\text {int }}$ for merging of 0.016 . The reflections with $F_{o}^{2}<0.2 \sigma\left(F_{0}^{2}\right)$ were reset to $F_{o}^{2}=$ $0.2 \sigma\left(F_{0}{ }^{2}\right)$. The $\sigma\left(F_{0}{ }^{2}\right)$ and $\sigma\left(F_{o}\right)$ values were defined as follows: $\sigma\left(F_{o}{ }^{2}\right)=\left[\sigma^{2} \text { count }+\left(0.02 F_{0}{ }^{2}\right)^{2}\right]^{1 / 2}$ and $\sigma\left(F_{o}\right)$ $=\sigma\left(F_{0}^{2}\right) / 2 F_{o}$. All reflections were used in subsequent calculations. The structure was solved by direct methods using MULTAN78 (Main et al., 1978). All $\mathrm{H}$ atoms were located from difference Fourier maps. Full-matrix refinement was carried out with anisotropic refinement for non- $\mathrm{H}$ atoms and isotropic refinement for $\mathrm{H}$ atoms. The function minimized was $\sum w\left(\left|F_{o}\right|-\left|F_{c}\right|\right)^{2}$ with $w=1 / \sigma\left(F_{o}\right)^{2}$. Refinement converged to $R=0.038, w R=0.064$ and $S=0.83$ for 1884 reflections. A final difference Fourier map was featureless $\left( \pm 0.15\right.$ e $\left.\AA^{-3}, \Delta / \sigma<0.34\right)$. Atomic scattering factors were from International Tables for $X$-ray Crystallography (1974, Vol. IV). All calculations were performed on a VAX8650 computer using the program KUDNA (Takusagawa, 1984). 
Table 2. Bond distances $(\AA)$ and angles $\left({ }^{\circ}\right)$

\begin{tabular}{|c|c|c|c|}
\hline$N(1)-C(2)$ & $1.370(4)$ & $C(14)-C(15)$ & $1.410(5)$ \\
\hline$N(1)-C(6)$ & $1.471(4)$ & $C(14)-C(19)$ & $1.386(5)$ \\
\hline$N(1)-C\left(1^{\prime}\right)$ & $1.454(4)$ & $C(15)-C(16)$ & $1.383(6)$ \\
\hline$C(2)-N(3)$ & $1.401(4)$ & $C(16)-C(17)$ & $1.389(8)$ \\
\hline $\mathrm{C}(2)-\mathrm{O}(2)$ & $1.210(4)$ & $C(17)-C(18)$ & $1.376(6)$ \\
\hline$N(3)-C(4)$ & $1.358(4)$ & $C(18)-C(19)$ & $1.399(5)$ \\
\hline$C(4)-C(5)$ & $1.493(5)$ & $C(19)-C(20)$ & $1.515(5)$ \\
\hline $\mathrm{C}(4)-\mathrm{O}(4)$ & $1.217(4)$ & $C\left(1^{\prime}\right)-C\left(2^{\prime}\right)$ & $1.532(5)$ \\
\hline$C(5)-C(6)$ & $1.529(4)$ & $C\left(1^{\prime}\right)-O\left(1^{\prime}\right)$ & $1.425(4)$ \\
\hline$C(5)-C(20)$ & $1.543(5)$ & $C\left(2^{\prime}\right)-C\left(3^{\prime}\right)$ & $1.515(5)$ \\
\hline$C(6)-C(7)$ & $1.531(5)$ & $\mathrm{C}\left(2^{\prime}\right)-\mathrm{O}\left(2^{\prime}\right)$ & $1.427(5)$ \\
\hline$C(6)-C(10)$ & $1.564(5)$ & $C\left(3^{\prime}\right)-C\left(4^{\prime}\right)$ & $1.529(5)$ \\
\hline$C(7)-C(8)$ & $1.519(6)$ & $C\left(3^{\prime}\right)-O\left(3^{\prime}\right)$ & $1.414(5)$ \\
\hline$C(8)-C(9)$ & $1.523(6)$ & $C\left(4^{\prime}\right)-C\left(5^{\prime}\right)$ & $1.519(6)$ \\
\hline$C(9)-C(10)$ & $1.525(5)$ & $\mathrm{C}\left(4^{\prime}\right)-\mathrm{O}\left(1^{\prime}\right)$ & $1.448(4)$ \\
\hline $\mathrm{C}(10)-\mathrm{N}(11)$ & $1.484(4)$ & $C\left(5^{\prime}\right)-O\left(5^{\prime}\right)$ & $1.426(6)$ \\
\hline$N(11)-C(12)$ & $1.467(5)$ & $\mathbf{C}\left(6^{\prime}\right)-\mathbf{C}\left(7^{\prime}\right)$ & $1.493(9)$ \\
\hline $\mathrm{N}(11)-\mathrm{C}(20)$ & $1.479(4)$ & $C\left(6^{\prime}\right)-C\left(8^{\prime}\right)$ & $1.480(8)$ \\
\hline$C(12)-C(13)$ & $1.543(5)$ & $\mathrm{C}\left(6^{\prime}\right)-\mathrm{O}\left(2^{\prime}\right)$ & $1.404(5)$ \\
\hline$C(13)-C(14)$ & $1.506(5)$ & $\mathrm{C}\left(6^{\prime}\right)-\mathrm{O}\left(3^{\prime}\right)$ & $1.426(6)$ \\
\hline$C(2)-N(1)-C(6)$ & $122.8(3)$ & $C(14)-C(15)-C(16)$ & 120.9 (4) \\
\hline$C(2)-N(1)-C(1)$ & $117.9(3)$ & $C(15)-C(16)-C(17)$ & $119.8(4)$ \\
\hline$C(6)-N(1)-C\left(1^{\prime}\right)$ & $118.6(3)$ & $C(16)-C(17)-C(18)$ & 119.1 (4) \\
\hline $\mathrm{N}(1)-\mathrm{C}(2)-\mathrm{N}(3)$ & $116.0(3)$ & $C(17)-C(18)-C(19)$ & $122.0(4)$ \\
\hline $\mathrm{N}(1)-\mathrm{C}(2)-\mathrm{O}(2)$ & $124.6(3)$ & $C(14)-C(19)-C(18)$ & $118.8(3)$ \\
\hline $\mathrm{N}(3)-\mathrm{C}(2)-\mathrm{O}(2)$ & $119.4(3)$ & $C(14)-C(19)-C(20)$ & $121.2(3)$ \\
\hline$C(2)-N(3)-C(4)$ & $126.1(3)$ & $C(18)-C(19)-C(20)$ & $120.0(3)$ \\
\hline$N(3)-C(4)-C(5)$ & $116.2(3)$ & $C(5)-C(20)-N(11)$ & $97.7(2)$ \\
\hline $\mathrm{N}(3)-\mathrm{C}(4)-\mathrm{O}(4)$ & $121.2(3)$ & $C(5)-C(20)-C(19)$ & $117.9(3)$ \\
\hline$C(5)-C(4)-O(4)$ & $122.7(3)$ & $N(11)-C(20)-C(19)$ & $113.2(3)$ \\
\hline$C(4)-C(5)-C(6)$ & $114.3(3)$ & $\mathrm{N}(1)-\mathrm{C}\left(1^{\prime}\right)-\mathrm{C}\left(2^{\prime}\right)$ & $116.5(3)$ \\
\hline$C(4)-C(5)-C(20)$ & $118.4(3)$ & $\mathrm{N}(1)-\mathrm{C}\left(1^{\prime}\right)-\mathrm{O}\left(1^{\prime}\right)$ & 110.1 (3) \\
\hline$C(6)-C(5)-C(20)$ & $105.2(3)$ & $\mathrm{C}\left(2^{\prime}\right)-\mathrm{C}\left(1^{\prime}\right)-\mathrm{O}\left(1^{\prime}\right)$ & $106.7(3)$ \\
\hline$N(1)-C(6)-C(5)$ & $110.6(3)$ & $C\left(1^{\prime}\right)-C\left(2^{\prime}\right)-C\left(3^{\prime}\right)$ & 104.9 (3) \\
\hline$N(1)-C(6)-C(7)$ & $112.4(3)$ & $C\left(1^{\prime}\right)-C\left(2^{\prime}\right)-O\left(2^{\prime}\right)$ & $109.3(3)$ \\
\hline $\mathrm{N}(1)-\mathrm{C}(6)-\mathrm{C}(10)$ & $113.2(3)$ & $\mathrm{C}\left(3^{\prime}\right)-\mathrm{C}\left(2^{\prime}\right)-\mathrm{O}\left(2^{\prime}\right)$ & $104.4(3)$ \\
\hline$C(5)-C(6)-C(7)$ & $112.8(3)$ & $C\left(2^{\prime}\right)-C\left(3^{\prime}\right)-C\left(4^{\prime}\right)$ & $106.4(3)$ \\
\hline$C(5)-C(6)-C(10)$ & $101.9(3)$ & $\mathrm{C}\left(2^{\prime}\right)-\mathrm{C}\left(3^{\prime}\right)-\mathrm{O}\left(3^{\prime}\right)$ & $104.3(3)$ \\
\hline$c(7)-C(6)-C(10)$ & $105.4(3)$ & $\mathrm{C}\left(4^{\prime}\right)-\mathrm{C}\left(3^{\prime}\right)-\mathrm{O}\left(3^{\prime}\right)$ & $111.1(3)$ \\
\hline$C(6)-C(7)-C(8)$ & $103.5(3)$ & $\mathrm{C}\left(3^{\prime}\right)-\mathrm{C}\left(4^{\prime}\right)-\mathrm{C}\left(5^{\prime}\right)$ & $113.9(3)$ \\
\hline$C(7)-C(8)-C(9)$ & $103.6(3)$ & $\mathrm{C}\left(3^{\prime}\right)-\mathrm{C}\left(4^{\prime}\right)-\mathrm{O}\left(1^{\prime}\right)$ & $106.2(3)$ \\
\hline$C(8)-C(9)-C(10)$ & $103.9(3)$ & $\mathrm{C}\left(5^{\prime}\right)-\mathrm{C}\left(4^{\prime}\right)-\mathrm{O}\left(1^{\prime}\right)$ & $105.9(3)$ \\
\hline$C(6)-C(10)-C(9)$ & $106.0(3)$ & $\mathrm{C}\left(4^{\prime}\right)-\mathrm{C}\left(5^{\prime}\right)-\mathrm{O}\left(5^{\prime}\right)$ & $112.7(3)$ \\
\hline$C(6)-C(10)-N(11)$ & $105.3(3)$ & $C\left(7^{\prime}\right)-C\left(6^{\prime}\right)-C\left(8^{\prime}\right)$ & $110.5(5)$ \\
\hline $\mathrm{C}(9)-\mathrm{C}(10)-\mathrm{N}(11)$ & $112.6(3)$ & $C\left(7^{\prime}\right)-C\left(6^{\prime}\right)-O\left(2^{\prime}\right)$ & $111.3(4)$ \\
\hline $\mathrm{C}(10)-\mathrm{N}(11)-\mathrm{C}(12)$ & $114.2(3)$ & $\mathrm{C}\left(7^{\prime}\right)-\mathrm{C}\left(6^{\prime}\right)-\mathrm{O}\left(3^{\prime}\right)$ & $107.6(4)$ \\
\hline$C(10)-N(11)-C(20)$ & $107.6(2)$ & $\mathrm{C}\left(8^{\prime}\right)-\mathrm{C}\left(6^{\prime}\right)-\mathrm{O}\left(2^{\prime}\right)$ & 112.1 (4) \\
\hline$C(12)-N(11)-C(20)$ & $111.8(3)$ & $\mathrm{C}\left(8^{\prime}\right)-\mathrm{C}\left(6^{\prime}\right)-\mathrm{O}\left(3^{\prime}\right)$ & $111.8(5)$ \\
\hline $\mathrm{N}(11)-\mathrm{C}(12)-\mathrm{C}(13)$ & $111.8(3)$ & $O\left(2^{\prime}\right)-C\left(6^{\prime}\right)-O\left(3^{\prime}\right)$ & $103.3(3)$ \\
\hline$C(12)-C(13)-C(14)$ & $110.7(3)$ & $\mathrm{C}\left(1^{\prime}\right)-\mathrm{O}\left(1^{\prime}\right)-\mathrm{C}\left(4^{\prime}\right)$ & $109.4(2)$ \\
\hline$C(13)-C(14)-C(15)$ & $118.3(3)$ & $\mathrm{C}\left(2^{\prime}\right)-\mathrm{O}\left(2^{\prime}\right)-\mathrm{C}\left(6^{\prime}\right)$ & $108.0(3)$ \\
\hline$C(13)-C(14)-C(19)$ & $122.6(3)$ & $\mathrm{C}\left(3^{\prime}\right)-\mathrm{O}\left(3^{\prime}\right)-\mathrm{C}\left(6^{\prime}\right)$ & $107.5(3)$ \\
\hline & & & \\
\hline
\end{tabular}

Discussion. Final fractional coordinates with equivalent isotropic temperature factors are listed in Table 1.* Table 2 contains molecular dimensions.

All ring systems are fused in a cis fashion (Fig. 1). The tetrahydroisoquinoline and the cyclopentane ring $[\mathrm{C}(6)-\mathrm{C}(7)-\mathrm{C}(8)-\mathrm{C}(9)-\mathrm{C}(10)]$ are trans to each other with respect to their connection to the pyrrolidine ring $[\mathrm{C}(5)-\mathrm{C}(6)-\mathrm{C}(10)-\mathrm{N}(11)-\mathrm{C}(20)]$ with the tetrahydroisoquinoline on the same side of the isopropylideneribose moiety. The absolute configurations for the three newly created chiral centers are $5 R, 6 R$ and $10 S$.

Understandably, the creation of two $s p^{3}$ centers $[\mathrm{C}(5)$ and $\mathrm{C}(6)]$ renders the uracil ring no longer

* Lists of anisotropic thermal parameters, H-atom parameters, complete molecular dimensions and structure factors have been deposited with the British Library Document Supply Centre as Supplementary Publication No. SUP 71078 (25 pp.). Copies may be obtained through The Technical Editor, International Union of Crystallography, 5 Abbey Square, Chester $\mathrm{CH} 12 \mathrm{HU}$, England. [C.IF reference: $\mathrm{HH} 1000$ ] planar. The six-membered ring system exhibits a twisted conformation. The effect of $\mathrm{C}(6)$ substitution in this compound is similar to that of the $\mathrm{C}(6)$ substitution without the saturation of the $C(5), C(6)$ double bond, namely, the molecule has a syn conformation with respect to the $\mathrm{C}\left(1^{\prime}\right)-\mathrm{N}(1)$ single bond. The glycosidic torsion angle $\mathrm{O}\left(1^{\prime}\right)-\mathrm{C}\left(1^{\prime}\right)-\mathrm{N}(1)-$ $\mathrm{C}(6), \chi_{\mathrm{CN}}$, is $245^{\circ}$ which is similar to torsion angles found in pyrimidine nucleosides and nucleotides with syn conformations (Yamagata et al., 1983, and references therein).

As has been found in other nucleosides, the ribose ring exhibits a puckered conformation. However, the $\mathrm{O}\left(\mathrm{l}^{\prime}\right)$ endo conformation of the ribose is unusual and its pseudorotation phase angle is $102.2^{\circ}$ (Altona \& Sundaralingam, 1972), which is outside of the range seen for most ribose nucleosides (Yamagata et al., 1983; Altona \& Sundaralingam, 1972, and references therein). This could be the result of severe steric crowding due to the presence of the cyclopentane $[C(6)-C(7)-C(8)-C(9)-C(10)]$ as well as the tetrahydroisoquinoline rings. The amplitude of the pucker, $\tau_{m}$, is $26.3^{\circ}$, significantly flattened compared with 6-methyluridine $\left[35^{\circ}(A), 37^{\circ}(B)\right]$ (Suck \& Saenger, 1972) and 6-methyldeoxyuridine $\left(31^{\circ}\right)$ (Birnbaum, Hruska \& Niemczura, 1980). The conformation around $\mathrm{C}(4)-\mathrm{C}(5)$ is gauche-gauche with the torsion angles $\varphi_{O O}=-65.6^{\circ}$ and $\varphi_{O C}=50.5^{\circ}$, which are similar to those of isopropylideneuridine (Katti, Seshadri \& Viswamitra, 1981), one form of 6-methyluridine ( $A$ form; Suck \& Saenger, 1972), 6-methyldeoxyuridine (Birnbaum, Hruska \& Niemczura, 1980), 6-cyanoisopropylideneuridine (Yamagata et al., 1983) and 5-bromoisopropylideneuridine (Gautham, Seshadri \& Viswamitra, 1983). An intramolecular hydrogen bond occurs between the $5^{\prime}$-hydroxyl $\mathrm{H}$ atoms of the ribose moiety and the $\mathrm{C}(2)$ carbonyl $\mathrm{O}$ atom.

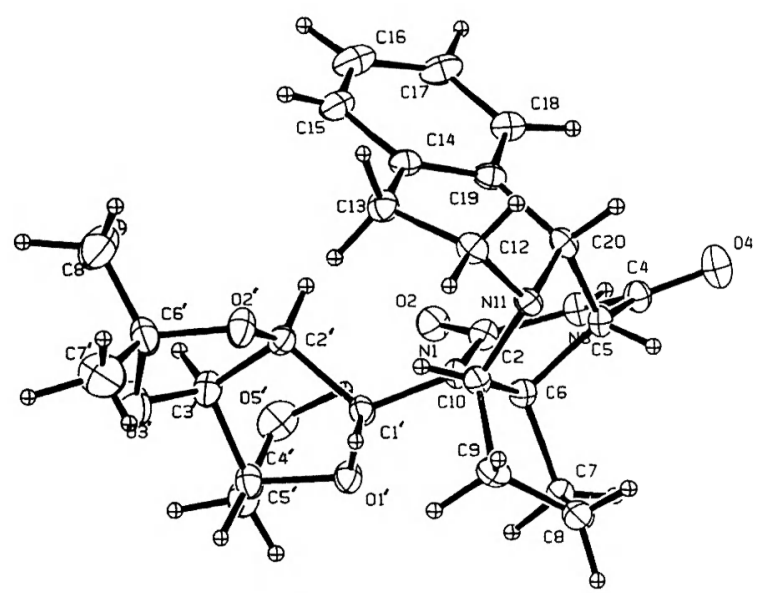

Fig. 1. ORTEP (Johnson, 1965) drawing of compound (1). 

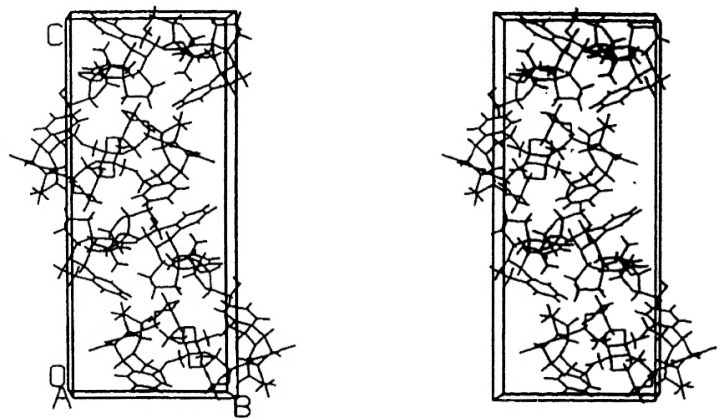

Fig. 2. Stereoscopic molecular packing diagram.

The dioxolane ring $\left[\mathrm{C}\left(2^{\prime}\right)-\mathrm{O}\left(2^{\prime}\right)-\mathrm{C}\left(6^{\prime}\right)-\mathrm{O}\left(3^{\prime}\right)-\right.$ $\left.\mathrm{C}\left(3^{\prime}\right)\right]$ has an envelope-like $\left[\mathrm{C}\left(6^{\prime}\right) /\right.$ exo $]$ conformation with an amplitude of pucker, $\tau_{m}$, of $36.4^{\circ}$. The pseudorotation phase angle is $166.3^{\circ}$.

Both the cyclopentane $[\mathrm{C}(6)-\mathrm{C}(7)-\mathrm{C}(8)-\mathrm{C}(9)-$ $\mathrm{C}(10), \mathrm{C}(8)-e x o]$ and pyrrolidine $[\mathrm{C}(5)-\mathrm{C}(6)-$ $\mathrm{C}(10)-\mathrm{N}(11)-\mathrm{C}(20), \quad \mathrm{C}(20)$-exo] rings have envelope conformations and exhibit high degrees of pucker (42.7 and $47.5^{\circ}$, respectively).

The molecular packing is shown in Fig. 2 and no intermolecular hydrogen bonds are observed.
This research has been supported by a Biomedical Research Support grant (RR5606) from the National Institutes of Health and a grant from the Wesley Foundation.

\section{References}

Altona, C. \& Sundaralingam, M. (1972). J. Am. Chem. Soc. 94, 8205-8212.

Birnbaum, G. I., Hruska, F. E. \& Niemczura, W. P. (1980). J. Am. Chem. Soc. 102, 5586-5590.

Gautham, N., Seshadri, T. P. \& Viswamitra, M. A. (1983). Acta Cryst. C39, 456-458.

JoHNSON, C. K. (1965). ORTEP. Report ORNL-3794. Oak Ridge National Laboratory, Tennessee, USA.

Katti, S. K., Seshadri, T. P. \& Viswamitra, M. A. (1981). Acta Cryst. B37, 407-410.

Main, P., Hull, S. E., Lessinger, L., Germain, G., DeclercQ, J.-P. \& Woolfson, M. M. (1978). MULTAN78. A System of Computer Programs for the Automatic Solution of Crystal Structures from X-ray Diffraction Data. Univs. of York, England, and Louvain, Belgium.

Suck, D. \& SAenger, W. (1972). J. Am. Chem. Soc. 94, 6520-6526.

Takusagawa, F. (1984). KUDNA. Crystallographic Computing System. Department of Chemistry, Univ. of Kansas, USA.

Wang, B., Mertes, M. P., Mertes, K. B. \& Takusagawa, F. (1990). Tetrahedron Lett. 31, 5543-5546.

Yamagata, Y., KobaYashi, Y., OKabe, N., Tomita, K., Sano, T., INOUE, H. \& UEDA, T. (1983). Nucleosides \& Nucleotides, pp. 335-343. 